\title{
EXPERIMENTAL STUDY OF THE TRANSPORT LIMITS OF INTENSE HEAVY ION BEAMS IN THE HCX ${ }^{1}$
}

\author{
L. R. Prost (FNAL), F. M. Bieniosek (LBNL), C.M. Celata (LBNL), A. Faltens (LBNL), \\ P. A. Seidl (LBNL), W. L. Waldron (LBNL) \\ R. Cohen (LLNL), A. Friedman (LLNL), M. Kireeff Covo (LLNL), S.M. Lund (LLNL), \\ A.W. Molvik (LLNL) \\ I. Haber (UM)
}

FNAL: Fermi National Accelerator Laboratory, Batavia, IL, 60510 USA

LBNL: Lawrence Berkeley National Laboratory, Berkeley, CA, 94720-8201

LLNL: Lawrence Livermore National Laboratory, Livermore, CA 94550 USA

UM: University of Maryland, College Park, MD 20742-3511

Corresponding author:

\section{Lionel Prost}

FERMILAB

P.O. Box 500 - MS 306

Batavia, IL, 60510 USA

Tel: $\quad 630-840-5614$

Fax: $\quad$ 630-840-4552

E-mail: $\quad$ lprost@fnal.gov

\begin{abstract}
The High Current Experiment (HCX) at Lawrence Berkeley National Laboratory is part of the US program to explore heavy-ion beam transport at a scale representative of the low-energy end of an induction linac driver for fusion energy production. The primary mission of this experiment is to investigate aperture fill factors acceptable for the transport of space-charge-dominated heavy-ion beams at high space-charge intensity (line charge density up to $\sim 0.2 \mu \mathrm{C} / \mathrm{m}$ ) over long pulse durations (4 $\mu \mathrm{s})$ in alternating gradient focusing lattices of electrostatic or magnetic quadrupoles. The experiment also contributes to the practical baseline knowledge of intense beam manipulations necessary for the design, construction and operation of a heavy ion driver for inertial fusion. This experiment is testing transport issues resulting from nonlinear space-charge effects and collective modes, beam centroid alignment and beam steering, matching, image charges, halo, electron cloud effects, and longitudinal bunch control. We first present the results for a coasting $1 \mathrm{MeV} \mathrm{K}^{+}$ion beam transported through the first ten electrostatic transport quadrupoles, measured with optical beam-imaging and double-slit phase-space diagnostics. This includes studies at two different radial fill factors (60\% and $80 \%)$, for which the beam transverse distribution was characterized in detail. Additionally, beam
\end{abstract}

\footnotetext{
${ }^{1}$ This work performed under the auspices of the U.S Department of Energy by University of California, Lawrence Livermore and Lawrence Berkeley National Laboratories under contracts No. W-7405-Eng-48 and DE-AC03-76SF00098.
} 
energy measurements will be shown. We then discuss the first results of beam transport through four pulsed room-temperature magnetic quadrupoles (located downstream of the electrostatic quadrupoles), where the beam dynamics become more sensitive to the presence of secondary electrons.

PACS numbers: 52.58.Hm, 52.59.Sa, 52.65.Rr, 41.85.Ne, 41.75.Ak, 52.70.Nc, 41.85.Lc

Keywords: inertial fusion energy, heavy-ion, linear accelerator, space-charge, phasespace, electrostatic transport, magnetic transport, quadrupole 


\section{INTRODUCTION}

The Heavy-Ion-Fusion (HIF) Program's objective is to provide a comprehensive scientific knowledge base for inertial fusion energy (IFE) driven by high-brightness heavyion beams. The High Current Experiment (HCX) [1] located at Lawrence Berkeley National Laboratory and carried out by the HIF-VNL, will explore beam transport limitations at a scale characteristic of the low-energy end of a multibeam induction linear accelerator driver, using a single beam.

At an injection energy of 1-1.8 MeV, a line-charge density, 1 , of $0.1-0.2 \mathrm{mC} \mathrm{m}^{-1}$ and a pulse duration of $4 \mathrm{~ms}$, the HCX main beam parameters are in the range of interest for a fusion driver front-end. At $1 \mathrm{MeV}$ where we performed our experiments, the generalized beam perveance is

$$
K=\frac{2 q I}{4 \pi \varepsilon_{0} m(\beta \gamma c)^{3}}=8 \times 10^{-4} .
$$

In this regime space-charge forces strongly influence the beam properties during its transport, and image charges induced on metallic structures of the machine aperture play an important role.

A principal goal of the HCX experiment is to evaluate the maximum acceptable beam fill factor, i.e. the maximum radial extent of the beam within the physical aperture, addressing the question of how compact a multiple-beam focusing lattice can be to accommodate the transport and acceleration of the heavy-ion beams. Higher fill factors are desirable because they make more economically efficient use of material structures. The fill factor study in this experiment addresses the more fundamental issue of how much charge can be transported in a single channel without deteriorating the beam quality. Greater fill factors enhance non-ideal physics effects resulting from imperfect focusing optics, image charges and halo, impacting material structures and releasing desorbed gases that interact with long-pulse beams creating possible electron-cloud effects. Design and engineering issues such as the frequency of correction elements and the alignment tolerances, are also less favorable the greater the fill factor and are addressed.

The initial configuration of HCX consisted of electrostatic quadrupoles, which provides efficient transport at low energy and provides clearing fields which sweep out unwanted electrons. However, magnetic transport experiments have commenced, to gain operational experience and to explore special limitations associated with magnetic focusing, in particular the onset of transport-limiting effects due to electrons trapped in the potential well of the ion beam.

\section{EXPERIMENT AND DIAGNOSTICS}

The present configuration consists of the $\mathrm{K}^{+}$ ion source and injector, an electrostatic quadrupole matching section (six quadrupoles), ten electrostatic transport quadrupoles and four room-temperature pulsed magnetic quadrupoles (Fig. 1).

[Figure 1 - two column width]

The ion source and injector system [2] delivers 1-1.8 $\mathrm{MeV} \mathrm{K}^{+}$ions, with corresponding ion beam current ranges from 0.2 to $0.6 \mathrm{~A}$. The emittance of the beam at the exit of the injector is $\mathrm{e}_{\mathrm{n}} \approx 1 \mathrm{p} \mathrm{mm} \mathrm{mrad}$.

The matching section consists of six electrostatic quadrupoles where the clear bore radii of the first (QM1) and last (QM6) are 100 $\mathrm{mm}$ and $31 \mathrm{~mm}$, respectively. The last three quadrupoles of the matching section (QM4-6) may each be displaced in the horizontal and vertical direction to correct for beam centroid offsets.

The electrostatic transport section consists of 10 quadrupoles ( $23 \mathrm{~mm}$ bore radius) on a common supporting rail [3]. The quadrupoles are aligned to within $\pm 100 \mathrm{~mm}$ on the bench before installation inside the vacuum tank. The last two quadrupoles (QI9 and QI10) may be displaced to steer the beam and correct betatron centroid oscillations.

The magnetic transport section consists of four room-temperature pulsed quadrupole magnets (MA1-MA4) with elliptical beam tubes [4]. They are mounted on a common support structure and are interfaced to one another with boxes that provide the drifts, pumping and diagnostic access in the alternating gradient lattice. The vacuum boxes also house electron clearing electrodes that consist of positively biased (up to $\approx 10 \mathrm{kV}$ ) annular stainless steel rings. The magnets are arranged such that the major radii of the apertures coincide with the focusing direction of the quadrupole field. The beam aperture semi-major and semi-minor radii are $5 \mathrm{~cm}$ and $3 \mathrm{~cm}$, respectively.

A multi-purpose diagnostic station (D-end) is at the end of the beam line. Beam diagnostics [5] are also located at the interface of the matching section and the electrostatic transport section (QD1) and after the last transport 
quadrupole in the electrostatic periodic lattice (D2). They include transverse slit scanners that measure in detail the transverse phase-space distribution of the beam, and Faraday cups and current transformers for total beam current measurements. Because the longitudinal space required by these diagnostics is greater than the available drift space between the focusing elements, the quadrupole at QD1 slides out of the beam path to allow for intercepting beam diagnostics measurements. The last two of the four quadrupole magnets are equipped with diagnostics designed to explore beam-gas and electron-cloud issues (e.g.: beam position monitors, flush probes, gridded probes, ion and electron energy analyzers) [6,7].

A Gas and Electron Source Diagnostic (GESD) [7] is located at the end of the diagnostics tank (D-end) and is used to measure electron emission and gas desorption yields from ions incident on targets near grazing incident ion angle. These data are intended for calibration of the signal intensities collected on the electrodes in the magnetic quadrupoles so that the beam loss and the gas desorption rate may be inferred. The GESD has also been used to study mitigation techniques for these effects.

An electrostatic energy analyzer (EA) is used for direct beam kinetic energy measurements but also provides time-dependent longitudinal phase-space information. The relative accuracy is $\pm 0.2 \%$, allowing detection of small energy variations as a function of time during the beam pulse.

Beam energy measurements can also routinely be done by a time-of-flight (TOF) technique [8]. A fast pulser (0.3 ms FWHM) induces $1 \%$ energy perturbations (space-charge waves) near the middle of the beam pulse at the first matching section quadrupole. These energy pulses manifest as 5-10\% current perturbations when measured $5.4 \mathrm{~m}$ downstream. They have been used as a time stamp for an accurate determination of the time of flight of the particles.

Additionally, a prototype scintillator-based optical diagnostic [9] is installed in the D-end tank and a more compact optical diagnostic subsequently developed is installed at D2. Their function is equivalent to the slit scanner with the additional advantage of providing information about the full 4-D transverse beam distribution rather than integrated slit projection, because intensity along the slit is also measured.
All the electrostatic quadrupoles are biased through coupling circuits, allowing them to act as capacitive pickups and beam loss monitors.

\section{ELECTROSTATIC TRANSPORT}

We have made two fill-factor measurements, for the envelope filling $\approx 60 \%$ and $\approx 80 \%$ of the available bore diameter in the 10 electrostatic quadrupoles. For each fill factor measurement, the transverse phase space of the beam was characterized in detail at the exit of the matching section. Each case required a different matching solution (i.e. different quadrupole voltages) in the matching section. Because the fill factor was changed by tuning the upstream beam to the matched beam conditions in the transport section for a lower focusing gradient, rather than by changing the current, the undepressed betatron phase advance per lattice period $\left(\mathrm{s}_{0}\right)$ for the two cases differ $\left(69^{\circ}\right.$ and $48^{\circ}$ for the $60 \%$ and $80 \%$ fill factor cases, respectively). The depressed phase advance per lattice period (s) due to the self potential of the beam are $13^{\circ}$ and $8^{\circ}$ for the $60 \%$ and $80 \%$ fill factor cases.

The matching section takes the beam exiting the injector and compresses it in area transversely by a factor of $\approx 25$ to produce the ( $2 \times$ RMS ) matched beam envelope parameters $a, a^{\prime}, b$ and $b^{\prime}$ necessary for transport in the periodic electrostatic lattice. Pickup signals from the quadrupole electrodes indicate that beam loss is $\leq 0.5 \%$ of the total beam current in the entire matching section, $\approx 1 / 10$ of which is due to beambackground gas interactions.

Due to upstream alignment errors and non uniformities in the beam current density distribution, the beam centroid exiting the injector is offset from the beam line axis by $1-2$ $\mathrm{mm}$ and 3-5 mrad. The centroid at QD1 is centered by mechanical translation of the three steering quadrupoles QM4-6 in the matching section. The required displacements are determined by calculating the single particle motion through these quadrupoles, and then solving for the quadrupole displacements needed to center the beam. By this procedure, the beam centroid positions $(\langle x\rangle,\langle y\rangle)$ and angles $\left(<x^{\prime}>, \quad<y^{\prime}>\right)$ are centered to within $0.5 \mathrm{~mm}$ and $2 \mathrm{mrad}$.

\section{[Figure 2]}

The horizontal phase-space of the beam measured at QD1 is shown in Fig. 2 for the 80\% fill factor case. In Fig. 2, the coherent envelope convergence of the beam has been removed to better show the details of the angular 
distribution. From the variance of more than 10 independent data sets at the diagnostics stations at the entrance and exit of the transport section and slightly different lattice gradients (i.e. various quadrupole voltage solutions in the matching and transport sections that resulted in the beam filling $60 \%$ or $80 \%$ of the clear aperture), the estimated emittance measurement uncertainty is $10 \%( \pm 1 \mathrm{~s})$. The systematic uncertainty is estimated to be $\approx \pm 4 \%$ (from sampling of the distribution and detector geometry accuracy). Within the uncertainties, the normalized emittance is nearly independent of the matching solution. The uncertainties for the beam envelope parameters were characterized by calculating the standard deviation (1s) of five repeated measurements, where the data was summed over a $1.5 \mathrm{~ms}$ window near the flat top region of the beam current pulse. By this measure, the stability and reproducibility of the envelope coordinate $(a, b)$ and angle $\left(a^{\prime}, b^{\prime}\right)$ measurements are $\approx 0.3 \mathrm{~mm}$ and $\approx 1 \mathrm{mrad}$, respectively.

In the entire length of the electrostatic transport section, the beam loss is $\approx 1 \%$ in midpulse for both fill factor cases. This is based on the sum of the currents collected on all 10 quadrupole electrodes and the ratio of Faraday cup currents at the entrance and exit of the transport section.

Within the experimental sensitivity, there is no evidence of emittance growth at the end of the electrostatic lattice for both the $60 \%$ and $80 \%$ fill factors in the horizontal plane. This result also holds for the vertical direction (diverging plane). Note also that the details of the beam phasespace distribution remain practically unchanged. PIC simulations initialized with semi-Gaussian distributions [10] have also predicted that matched beam excursions filling $80 \%$ of the quadrupole bore would result in negligible emittance growth, assuming perfect alignment and envelope control. However, these simulations do not include non-ideal effects resulting from particle loss. To date, comparisons of measured phase-space distributions to PIC simulations show that the measured distributions are not well reproduced in the theoretical model, even when initialized with a distribution reconstructed from the data [11].

Integrating the envelope equation from QD1 to D2 (initialized with QD1 measurements of envelope radii, convergence angles, current, and measurements of beam energy) gives a calculated envelope in agreement with the experiment at the D2 location to within $0.4 \mathrm{~mm}$ and $3 \mathrm{mrad}$. This level of agreement allows us to confidently rely on envelope model predictions to tune the lattice and control the beam envelope excursions in the experiment and implies that image forces do not significantly influence the RMS parameters. In Table I, envelope measurements at the exit of the electrostatic lattice for an $80 \%$ fill factor data set are compared to predictions of the envelope model. Envelope model uncertainties are taken from the standard deviation of a Monte Carlo distribution of envelope predictions through the transport section, where several thousand envelopes are calculated with initial conditions randomly distributed about the measured values. The initial distributions for the parameters that are varied are Gaussian with standard deviations representing the measurement uncertainties or the equipment accuracies (e.g.: stability of the quadrupole voltages).

[Table I]

Thus, Table I shows that the RMS envelope model is accurate to within the measurement uncertainty.

Defining the envelope mismatch as $\delta_{M}=\operatorname{Max}\left(a_{\text {Max }}, b_{\text {Max }}\right)-R_{0, \text { Max }}, \quad$ where $\operatorname{Max}\left(a_{\text {Max }}, b_{\text {Max }}\right)$ is the maximum of the envelope excursions in both planes of the calculated envelope initialized with QD1 measurements, and $R_{0, \text { Max }}$ is the maximum excursion for the theoretical matched beam, for both fill factor cases, we were able to match the beam to within $\delta_{M}=1 \pm 0.5 \mathrm{~mm}$. The uncertainty in $\delta_{M}$ is based on the Monte Carlo analysis discussed above.

We observe a diamond-shaped beam pattern at QD1 and at D2 (Fig. 3a), which is attributed to nonlinear fields arising from the space-charge component of the distribution and the collective evolution of the distribution in the ESQ injector and in the matching and transport sections. All the $J(x, y)$ data are used with the phase space data at QD1 to construct a consistent particle distribution for simulation studies [12].

The time-resolved crossed-slit data show that at QD1 the profile of the beam during the rise and fall of the beam current pulse is larger than during the flattop. Ballooning of the beam head was predicted for the beam exiting the injector from 3-D particle-in-cell simulations and is attributed to a voltage rise time in the diode which is presently too slow to match the space- 
charge field evolution of the beam head $[13,14]$. Calculations of the head-tail dynamics through the rest of the HCX are underway.

\section{MAGNETIC TRANSPORT}

After commissioning the quadrupole magnets, we are able to transport the beam with minimal beam loss $(<2 \%$, based on ratios of Faraday cup currents at the entrance and exit of the magnetic transport section). Earlier difficulties were attributed to large centroid betatron oscillations resulting from too strong gradients in the magnets, whose lattice period is twice as large as that of the electrostatic transport section upstream. In addition, the first generation of diagnostics in the third and fourth magnets reduced the available clear bore radius to $R=27$ $\mathrm{mm}$. These were replaced with a second generation of diagnostics whose shapes match more closely the elliptical bore of the magnets.

However, measurements of the beam current distribution with the optical diagnostic at D-end showed a very distorted distribution (Fig 3c) and filamentation of the phase-space as revealed by the single-slit image in Fig. 3b.

\section{[Figure 3]}

This effect was traced to the intercepting slit generating copious electrons in a field-free region. An electron suppressor placed between the end of the last transport magnets and the measuring plane, and biased negatively to a few $\mathrm{kV}$ prevents the electrons from rushing upstream. As a result, the phase-space does not filament and the beam distribution is not as distorted. In addition to the electron suppressor, electron clearing electrodes (steel annulus biased positive) were added to the lattice between the magnets. Their role is to collect secondary electrons that may be created in the magnets when the beam scrapes. Because the driver-scale beam dynamics are so sensitive to space-charge, these perveance-neutralizing effects can have a large impact on the transverse phase-space distribution.

The beam phase-space distribution was measured for the full beam current $(175 \mathrm{~mA})$ (Fig 4a) and for the beam going through an aperture located at $\mathrm{D} 2$ reducing its size and current (32 mA) (Fig 4b) to allow for more clearance in the magnets.

\section{[Figure 4]}

In Fig. 4a, the horizontal phase-space distribution for the full beam is very distorted, and accordingly, the measured emittance increased by a factor of four with respect to the upstream measurement. However, the vertical phase-space distribution shows little distortion and the emittance does not grow. For the apertured beam, although its phase-space distribution appears slightly distorted at the exit of the magnetic transport section, negligible emittance growth is observed.

PIC simulations initialized with envelope measurements at D2 and measured quadrupole moment z-dependence, agree well with the envelope measurements at $\mathrm{D}$-end for the apertured beam, but the agreement remains poor for the full beam. In addition, the large emittance measured in the horizontal direction at D-end for the full beam has not been reproduced by simulation.

\section{BEAM ENERGY MEASUREMENTS}

The EA measurements show a $10 \%$-higher energy head and long low-energy tail (Fig. 5), which are due to longitudinal space charge that accelerates particles at the head and decelerates particles at the tail of the pulse, and the extraction voltage pulse shape. The beam mean energy is constant to within $0.5 \%$ for $3.1 \mathrm{~ms}$. Both the TOF and EA diagnostics determine the absolute beam energy to $\pm 2 \%$, with both measurements agreeing within these uncertainties.

\section{[Figure 5]}

From the energy analyzer, the beam mean energy is $962 \mathrm{keV}$. The precise determination of the beam energy is essential for the agreement between envelope simulations and experimental data (Sec. 3).

\section{CONCLUSIONS}

The High Current Experiment studies how much clearance is needed to transport high-linecharge-density, high-perveance beams with acceptable beam degradation. This is important input for the future design of a heavy-ion fusion driver, which has an array of beams.

The results of transport through the first ten electrostatic quadrupoles indicate transport with a high fill factor (at least $80 \%$ ) is possible with negligible emittance growth and acceptable beam loss near the front-end of a driver. This was achieved in a single beam line that includes most of the uncertainties that surround the construction of a full-scale driver (e.g.: manufacturing of components, system alignment, high voltage stability) and the production of a high current beam (current density nonuniformities, velocity tilt, halo particles, partial neutralization) except for the uncertainties 
related to a multibeam array configuration. Details of the measured phase space distribution are being used to initialize particle-in-cell simulations for comparison of data with theoretical models. While 10 or 20 quadrupoles are too few for settling questions of emittance evolution in a long transport system, they are of the correct length for the rapid initial evolution of the emittance and beam profile, which is expected in the front end of an accelerator.

The beam energy distribution was measured and is consistent with earlier measurements [15], where the head was found to be accelerated and the tail decelerated with respect to the flattop of the beam. This effect is due to the longitudinal space-charge of the beam and is understood theoretically.

Preliminary measurements in the magnetic transport section showed beam loss and accompanied large distortions of the beam current density with the first generation of electron diagnostics inside the magnets. A better choice of the magnet gradients and redesigned magnet diagnostics allowed more clearance to the wall and permitted the transport of the full beam without loss. These difficulties are partly attributed to the fact that the magnetic lattice is very different from the upstream electrostatic lattice. The fast transition between the two sections has dynamical effects that were not captured in early calculations, which only employed a simple envelope model. In addition, it is clear that even a little scraping of the beam will generate enough secondary electrons to affect the beam dynamics more strongly than in the electrostatic transport, where they are swept away. Quantitative measurements of the secondary electrons, ions and atoms in magnetic quadrupoles have started.

Future plans include an integrated beam transport experiment with $\approx 100$ quadrupoles such as described in Ref. $[16,17,18]$, designed to explore transverse phase-space dynamics as well as longitudinal phase-space dynamics during compression and final focusing, integrating for the first time several beam physics manipulations required in a fusion driver.

\section{ACKNOWLEDGEMENTS}

We are grateful to R. Hipple, W. Strelo and their staff for excellent technical support.

\section{REFERENCES}

[1] P.A. Seidl et al., in Proceedings of the 2001 Particle Accelerator Conference, Piscataway, 2001, edited by Peter Lucas and Sara Weber, (IEEE, Piscataway, NJ, 2001), p.2932.

[2] J.W. Kwan, F. M. Bieniosek, E. Henestroza, L. Prost, P. Seidl, Laser and Particle Beams 20(3), 441 (2002).

[3] P. A. Seidl et al., Laser and Particle Beams 20(3), 435 (2002)

[4] P. A. Seidl et al., these proceedings (Th.P.22).

[5] F. M. Bieniosek et al., these proceedings (Th.I.10).

[6] A. W. Molvik et al., these proceedings (Th.I.01).

[7] A. W. Molvik et al., Phys. Rev. ST-AB (to be published)

[8] F. M. Bieniosek et al., these proceedings (Th.P.21).

[9] F. M. Bieniosek, L. Prost and W. Ghiorso, in Proceedings of the 2003 Particle Accelerator Conference, Portland, 2003, edited by Joe Chew, Peter Lucas, and Sara Webber, (IEEE, Piscataway, NJ, 2003), p. 2524.

[10] C. M. Celata, D. P. Grote and I. Haber, Laser and Particle Beams 20(4), 577 (2002).

[11] A. Friedman et al., in Proceedings of the 2003 Particle Accelerator Conference, Portland, 2003, edited by Joe Chew, Peter Lucas, and Sara Webber, (IEEE, Piscataway, NJ, 2003), p. 275.

[12] A. Friedman, D. P. Grote, C. M. Celata and J. W. Staples, Laser and Particle Beams 21(1), 17 (2003).

[13] M. Lampel and M. Tiefenback, Appl. Phys. Lett. 43(1), 57 (1983).

[14] J.-L. Vay et al., Phys. Plasmas, in press.

[15] S.S. Yu et al., in Proceedings of International Symposium on Heavy Ion Inertial Fusion, Princeton, 1996, edited by J. J. Barnard, T. J. Fessenden and E. P. Lee (PPPL, Princeton, NJ, 1996), p. 309.

[16] M. A. Leitner et al., in Proceedings of the 2003 Particle Accelerator Conference, Portland, 2003, edited by Joe Chew, Peter Lucas, and Sara Webber, (IEEE, Piscataway, NJ, 2003), p. 1521.

[17] J. J. Barnard et al., Laser and Particle Beams 21(4), 553 (2003).

[18] C. M. Celata et al., in Proceedings of the Third International Conference on Inertial Fusion Sciences and Applications (IFSA 2003), Monterey, CA, Sept. 7-12, 2003. LBNL-52409. 


\section{Figures and Tables captions:}

Figure 1: Layout of the HCX (elevation view).

Figure 2: Horizontal phase-space diagrams (a) before and (b) after the electrostatic transport section for the $80 \%$ fill factor case $(\mathrm{Dt}=0.12 \mathrm{~ms}$ at mid-pulse).

Figure 3: Images of the beam acquired with the optical diagnostic (1 ms gate width). (a) Sum of slit images at D2; (b)-(c) Single-slit image and sum of slit images, respectively, at D-end before installation of the electron suppressor; (d) Sum of slit images at D-end after installation of the electron suppressor. The horizontal dark bands are due to supporting bridges on the measuring slit.

Figure 4: Horizontal phase-space diagrams before (top) and after (bottom) the magnetic transport section for (a) the full beam (175 mA), (Dt $=0.52 \mathrm{~ms}$, corresponding to the flattest part of the beam pulse); (b) the apertured beam $(32 \mathrm{~mA}),(\mathrm{Dt}=1.2 \mathrm{~ms}$ at mid-pulse $)$.

Figure 5: Longitudinal energy distribution measured with the electrostatic energy analyzer. $\mathrm{K}^{+}$ions were detected at the beam head with $1.1 \mathrm{MeV}$ (not visible on this scale).

Table I: Experimental envelope parameters compared to envelope model predictions at the exit of the electrostatic section. The data are from a $120 \mathrm{~ns}$ interval of the flattop region of the beam pulse. 


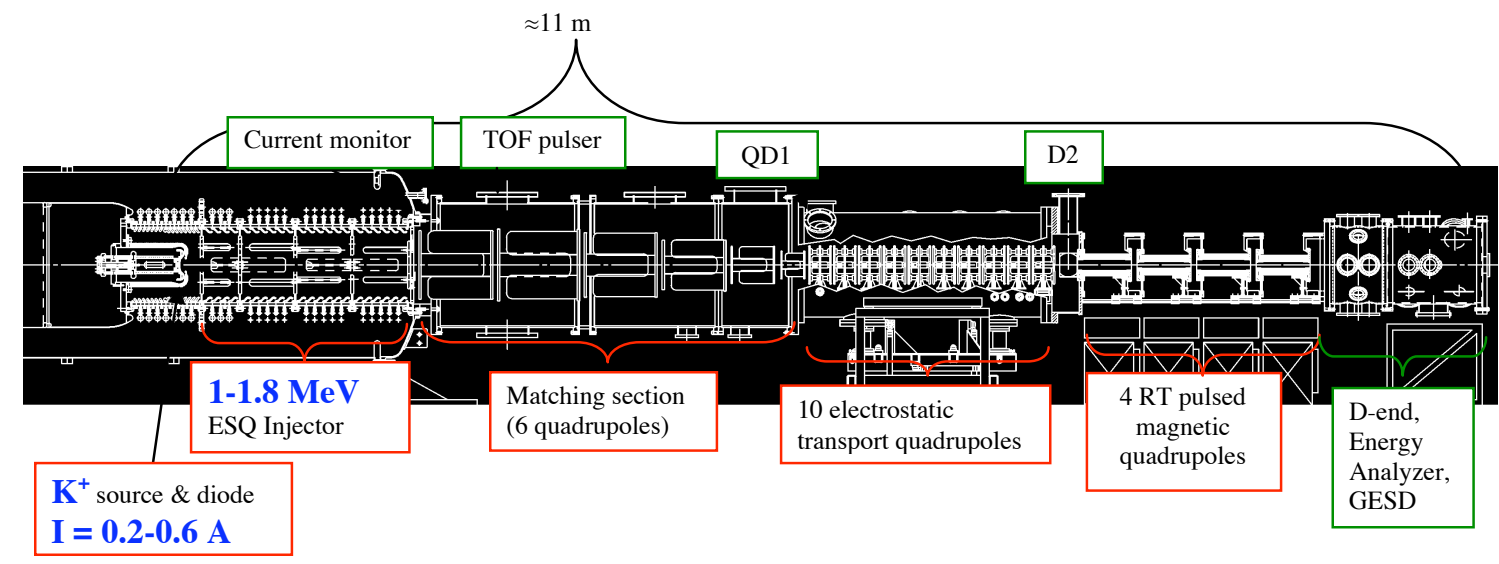

Figure 1: (Two column width) Layout of the HCX (elevation view). 


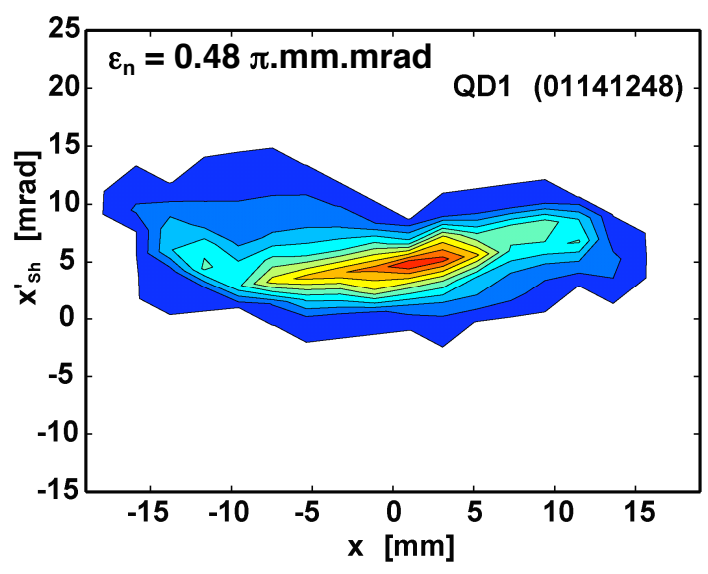

(a)

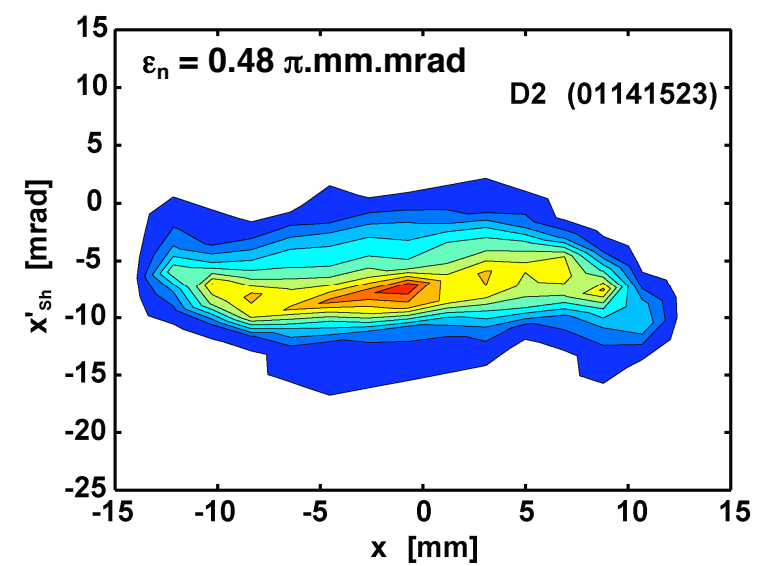

(b)

Figure 2: Horizontal phase-space diagrams (a) before and (b) after the electrostatic transport section for the $80 \%$ fill factor case $(\mathrm{Dt}=0.12 \mathrm{~ms}$ at mid-pulse $)$. 


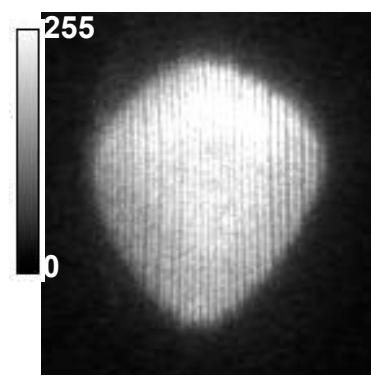

(a)

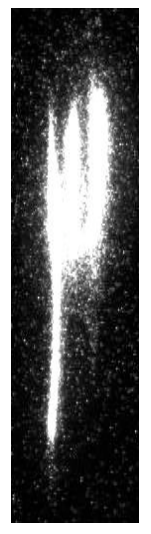

(b)

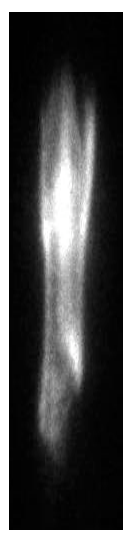

(c)

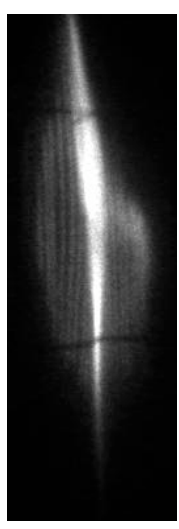

(d)

Figure 3: Images of the beam acquired with the optical diagnostic (1 ms gate width). (a) Sum of slit images at D2; (b)-(c) Single-slit image and sum of slit images, respectively, at D-end before installation of the electron suppressor; (d) Sum of slit images at D-end after installation of the electron suppressor. The horizontal dark bands are due to supporting bridges on the measuring slit. 

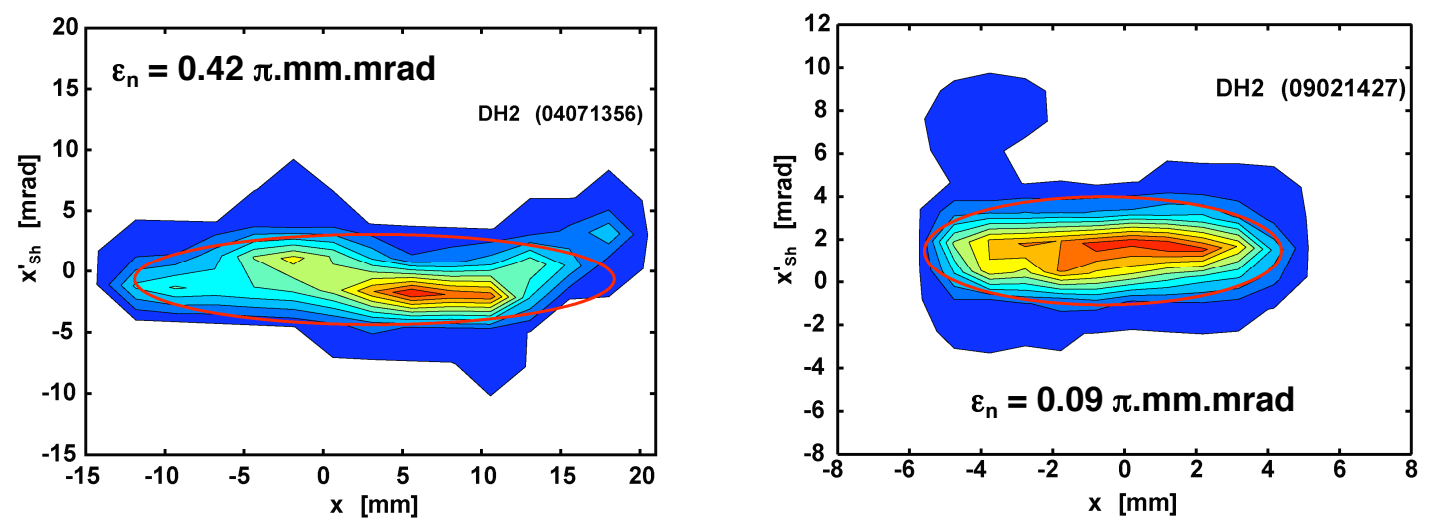

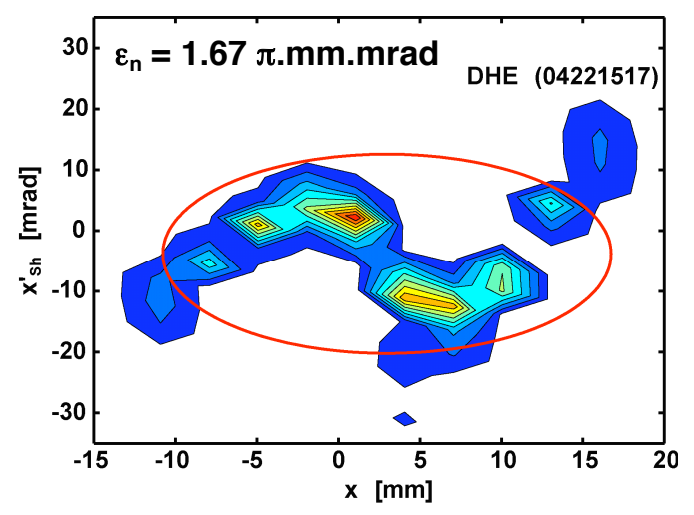

(a)

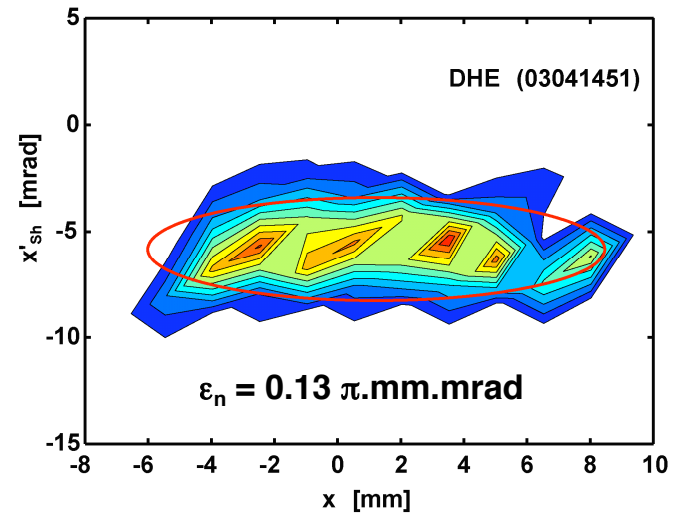

(b)

Figure 4: Horizontal phase-space diagrams before (top) and after (bottom) the magnetic transport section for (a) the full beam (175 mA), ( $\mathrm{Dt}=0.52 \mathrm{~ms}$, corresponding to the flattest part of the beam pulse); (b) the apertured beam ( $32 \mathrm{~mA}),(\mathrm{Dt}=1.2 \mathrm{~ms}$ at mid-pulse). The sharp structures ('islands') in the distributions are an artifact of the contour algorithm. 


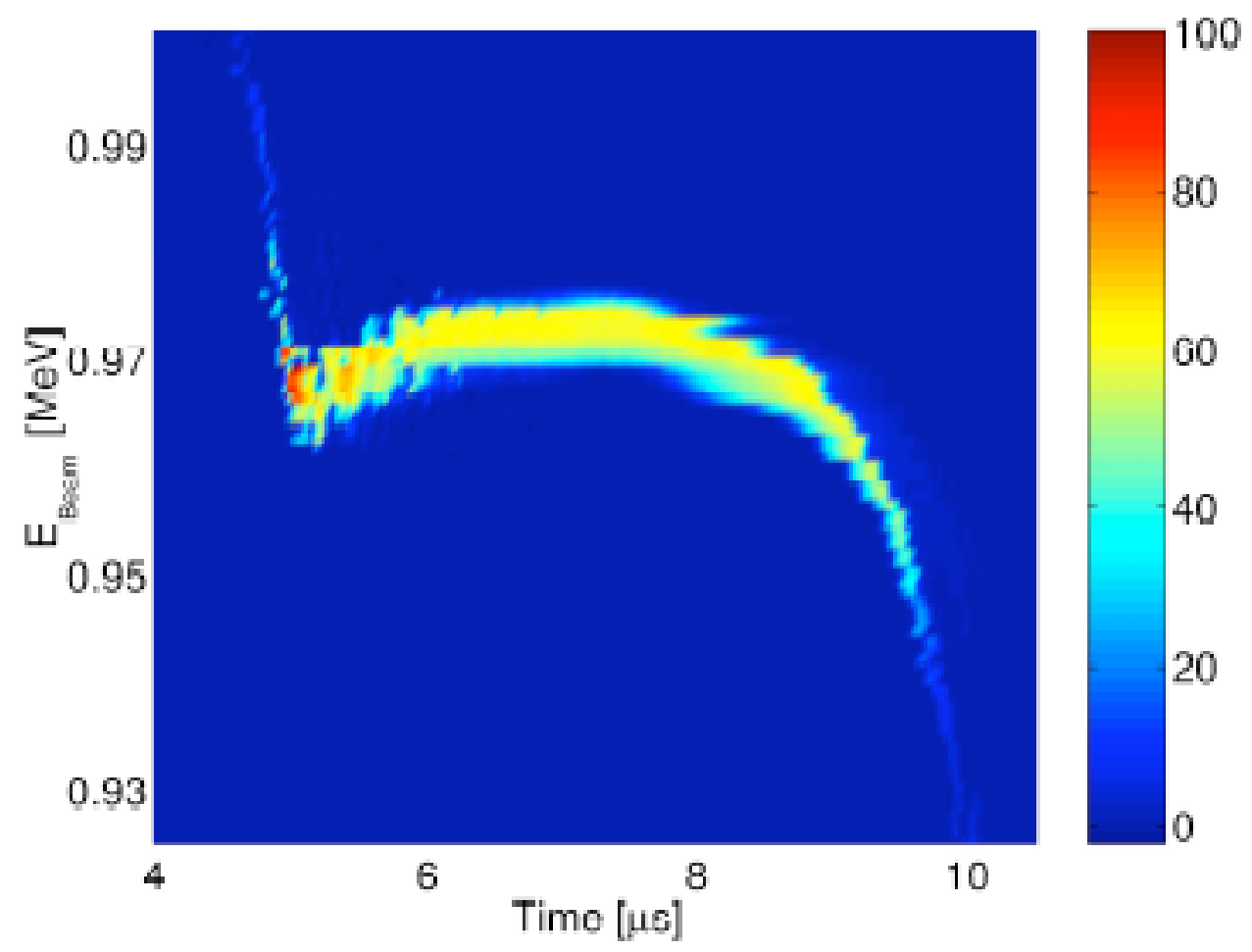

Figure 5: Longitudinal energy distribution measured with the electrostatic energy analyzer. $\mathrm{K}^{+}$ions were detected at the beam head with $1.1 \mathrm{MeV}$ (not visible on this scale). 
Table I: Experimental envelope parameters compared to envelope model predictions at the exit of the electrostatic section. The data are from a $120 \mathrm{~ns}$ interval of the flattop region of the beam pulse.

\begin{tabular}{cccccc}
\hline \hline & & $\begin{array}{c}\mathrm{a} \\
{[\mathrm{mm}]}\end{array}$ & $\begin{array}{c}\mathrm{a}_{-} \\
{[\mathrm{mrad}]}\end{array}$ & $\begin{array}{c}\mathrm{b}^{2} \\
{[\mathrm{~mm}]}\end{array}$ & $\begin{array}{c}\mathrm{b}_{-} \\
{[\mathrm{mrad}]}\end{array}$ \\
\hline \multirow{2}{*}{$80 \% \mathrm{ff}$} & Exp. & $\mathbf{1 4 . 0 7}$ & $\mathbf{- 3 8 . 5 0}$ & $\mathbf{1 5 . 5 4}$ & $\mathbf{3 9 . 8 4}$ \\
& Model. & $\mathbf{1 4 . 6 6}$ & $\mathbf{- 3 8 . 0 5}$ & $\mathbf{1 5 . 1 3}$ & $\mathbf{3 8 . 1 0}$ \\
$\begin{array}{c}\text { Uncertainty } \\
( \pm 1 \sigma)\end{array}$ & Exp. & $\mathbf{0 . 3}$ & $\mathbf{1 . 0}$ & $\mathbf{0 . 3}$ & $\mathbf{1 . 0}$ \\
\hline \hline
\end{tabular}

